\title{
Pérdida y Afrontamiento en Desempleados: Adaptación del Inventario Texas Revisado de Duelo a la Situación de Pérdida de Empleo
}

\author{
Loss and Coping in Unemployed: Adaptation of the Texas Revised Inventory of \\ Grief to the Situation of Loss of Employment
}

\author{
Carmen Díaz ${ }^{1}$, Yolanda Navarro Abal $^{1}$, José Antonio Climent ${ }^{1}$, Elena Ortega ${ }^{1}$, María José López- \\ López ${ }^{1}$ y Jesús Casado ${ }^{1}$
}

\begin{abstract}
Resumen
Este estudio aplica por primera vez el Inventario Texas Revisado de Duelo (ITRD) en un contexto de pérdida de empleo. Los participantes de este estudio son 217 desempleados de la ciudad de Huelva. Se realiza un análisis de los ítems del instrumento obteniendo una fiabilidad de consistencia interna de .85 y .90 en cada parte del instrumento. El análisis factorial exploratorio extrae dos factores coincidentes con el cuestionario original. Con objeto de obtener evidencias de validez se realiza un análisis de conglomerados de dos fases, mostrando la agrupación de las personas en dos conglomerados, uno relacionado con duelo prolongado y el otro con intensidad menor. No se encuentran diferencias respecto a la edad y el sexo en la intensidad de duelo, pero si respecto a la antigüedad en desempleo y responsabilidad sobre los ingresos familiares. Se encuentra un afrontamiento evitativo en personas que presentan un duelo más intenso.
\end{abstract}

Palabras clave: duelo, desempleo, afrontamiento, evaluación

\begin{abstract}
This study firstly apply the Texas Revised Inventory of Grief (TRIG) in a context of job loss. Participants in this study were 217 unemployed in the city of Huelva. An items analysis and internal consistency reliability was computed obtaining alpha coefficients of .85 and .90 in each part of the instrument. The exploratory factor analysis extracted two factors similar to those in the original questionnaire. In order to obtain validity evidence, cluster analysis in two stages was performed, showing that participants fitted into two clusters, one associated with prolonged and the other with less intensity grief. No differences regarding age and sex in the intensity of grief was found, but regarding the length of unemployment and responsibility on the family income. An avoidant coping style was found in people with more intense grief.
\end{abstract}

Keywords: coping, grief, unemployment, assessment

\footnotetext{
${ }^{1}$ Doctora en Psicología. Universidad de Huelva. Departamento de Psicología Clínica, Experimental y Social. Facultad de Ciencias de la Educación. Campus del Carmen, Avenida de las Fuerzas Armadas s/n. 21117 Huelva, España. Correo: carmen.diaz@dpsi.uhu.es, 959218428
} 


\section{Introducción}

Cada vez es más frecuente que la persona se encuentre desempleada en diversos momentos de su vida laboral. El desempleo implica una serie de consecuencias físicas, sociales y psicológicas de gran relevancia (Buendía, 2010; Karsten \& Moser, 2009). Estudios clásicos realizados en Europa y en Estados Unidos indican que las dificultades económicas son el resultado más frecuente del desempleo y que estas dificultades influyen en el bienestar psicosocial (Blanch, 1990; Buendía, 1990). La pérdida de empleo desencadena un proceso de reajuste a nivel global en la persona en todas sus dimensiones, personal, social y familiar (Afonso \& Poeschl, 2006). El desempleo supone la pérdida del contacto con una situación con la que se mantiene un vínculo afectivo. De esta forma, se produce una elaboración similar a la propia del duelo en cualquier otra circunstancia, como la pérdida de un ser querido.

El término duelo hace referencia al dolor experimentado ante la pérdida de un ser querido. Autores como Tizón (2004) lo entienden como un conjunto de procesos psicológicos y psicosociales que aparecen tras la pérdida de una persona con la que se estaba psicosocialmente vinculada. El manual diagnóstico DSM-V (APA, 2013) lo incluye en problemas que pueden ser objeto de atención clínica, codificándose como Z63.4 Duelo (V62.82). Por su parte, la clasificación internacional de las enfermedades CIE-10 (OMS, 2003) sólo incluye en los trastornos adaptativos F.43.2 las reacciones que, por su duración excesivamente prolongada en el tiempo y manifestaciones atípicas, se consideran anormales.

A lo largo de la historia, se exponen muchas concepciones diferentes del duelo, inicialmente basadas en una concepción pasiva. Siguiendo a Munera (2013), algunas teorías basan la explicación del duelo en función a la ansiedad de separación. Otros autores conciben el duelo como consecuencia de los apegos (Bowlby, 1980). También, Bowlby (1980) formula la teoría Biológica del duelo en la que se realiza una comparación entre los seres humanos y los animales en cuanto a reacciones emocionales relacionadas con procesos biológicos primitivos. Posteriormente se comenzó a analizar el duelo como proceso con un comienzo, desarrollo y final (Ortego \& Alconero, 2001). Diversos autores han representado modelos en los que se identifican las fases del duelo como proceso (Kübler-Ross, 1993; Sánchez \& Martínez, 2014). Autores como Fernández, Rodríguez y Diéguez (2006) critican esta visión del duelo por fases, entendiendo que es una percepción pasiva y de poco control en el proceso del duelo por parte del doliente. Para ellos, el afrontamiento del duelo requiere de una actitud activa. Estos modelos establecen que el duelo está influenciado por múltiples causas tanto individuales como sociales, y de esta forma, es muy difícil determinar sus etapas.

Otra de las cuestiones abordadas en la literatura en relación con el duelo guarda relación con las tipologías del mismo. En función a las características y objetivos de estudio de este trabajo, se sintetiza el modelo presentado por Neimeyer (2002), que distingue entre:

- Duelo anticipado: experimentado antes de una pérdida significativa. Puede considerarse como un duelo preparatorio para anticipar la pérdida.

- Duelo retardado o inhibido: se produce cuando las personas inicialmente no manifiestan conductas y sentimientos característicos de la tristeza generada por la pérdida, aparentando total normalidad. Este duelo es especialmente patogénico, al usar la persona la estrategia de afrontamiento de negación.

- Duelo prolongado: se mantiene durante un largo período de tiempo, mostrando la persona comportamientos que intentan mantener al ser querido vivo mediante su propio sufrimiento.

- Duelo complicado: en esta ocasión se acentúa todos los procesos típicos de un duelo normal, tales como culpabilidad, ideas irracionales, etc. que favorecen la somatización y no permiten la evolución del duelo ni la intervención del mismo durante un tiempo más o menos prolongado.

En cuanto a los factores de protección, tal como indican Stroebe, Folkman, Hansson, \& Schut (2006), entre los factores más estudiados se encuentran las creencias religiosas y el apoyo social (Álvaro \& Garrido, 2003; Villacieros, Serrano, Bermejo, Magaña, \& Carabias, 2014). Otras variables que se encuentran actualmente en 
el centro de atención de los investigadores se sitúan en la resiliencia (Gillham \& Seligman, 1999), crecimiento postraumático, personalidad fuerte o hardness (Tedeschi \& Kilmer, 2005).

En relación con los factores de riesgo de duelo específico, Sanders (1999), pone como ejemplo las características de la pérdida, siendo diferentes si la muerte es esperada o inesperada. Hegewald y Crapo (2007) señalan que variables como el nivel socioeconómico están relacionadas con la salud en general pero no existen datos suficientes para confirmar que module los efectos del duelo.

Stroebe et al. (2006) plantean un modelo en el que integran la teoría cognitiva del estrés de Lazarus y Folkman (1984) y el modelo del proceso dual para el afrontamiento al duelo de Stroebe y Schut (1999). Cobra aquí relevancia las estrategias y estilos de afrontamiento como factor de riesgo y protección ante situaciones de duelo (Siracusa, Quintana, Marfil, Caro, Río-Valle, \& Martínez, 2011). Según Fernández-Abascal (1997) es importante distinguir entre estilos de afrontamiento, o predisposiciones personales para hacer frente a las situaciones; y las estrategias de afrontamiento que se utilizan en cada contexto y que dependen de las condiciones que la desencadenen. Meléndez, Mayordomo, Sancho y Tomás (2012), señalan variables como la edad o el género como variable moduladora del empleo de las diferentes estrategias de afrontamiento. Izquierdo-Rus y López-Martínez (2013) apuntan a la creatividad como una herramienta que maximiza las posibilidades de producir ideas novedosas y adecuadas para afrontar la situación de desempleo.

Habitualmente el estudio del duelo ha estado centrado en la pérdida de un ser querido. Se han desarrollado diferentes instrumentos de evaluación de la intensidad de duelo ante el fallecimiento de una persona cercana, como son el Inventario de Duelo Complicado (Prigerson et al., 1999), el Inventario de Experiencias en Duelo (Sanders, Mauger, \& Srong, 1985) o el el Inventario Texas Revisado de Duelo (The Texas Revised Inventory of Grief, ITRD, Faschingbauer, Zisook, \& De Vaul, 1987), siendo éste último uno de los más utilizados en la práctica clínica. La medición del duelo se hace fundamental también desde la perspectiva de la expresión emocional de las personas que pasan por estas situaciones, siendo esta expresión una forma de regular las emociones y afrontar la situación (Martínez-Pérez \& Sánchez-Aragón, 2011)

Adicionalmente, el término duelo puede también aplicarse a aquellos procesos psicológicos y psicosociales que se ponen en marcha ante cualquier tipo de pérdida (fracasos escolares, divorcio, problemas familiares, cambios de domicilio, problemas económicos, diagnóstico de una enfermedad grave, entre otros). GonzálezCalvo (2005) señala que los movimientos migratorios, en tanto que suponen un proceso de reorganización y adaptación a las nuevas claves socioculturales, conllevan una elaboración de duelo. También se aprecia como el proceso de duelo por separación (Cáceres, Manhey, \& Vidal, 2009), por duelo amoroso (Sánchez-Aragón \& Retana-Franco, 2013) o también, el síndrome del nido vacio, que se define como un período transitorio en donde se perciben determinados sentimientos consecuencia de la pérdida del rol desempeñado como madre o padre (Lugonés, 2001). Carmona, Cely, Nemeguen, \& Sanabria (2008) señalan que la jubilación marca el fin de una actividad laboral que sirvió para construir un rol social determinado con actividades y hábitos de vida y de pertenencia a grupos de identidad que desaparecerán.

Todas estas situaciones pueden originar reacciones desadaptativas con manifestaciones como tristeza, llanto, desesperanza, impotencia, rabia y culpa, además de disfunción social y laboral. De esta forma, es evidente que el duelo se elabora en cualquier ámbito de la vida en la que se produce una pérdida o un cambio relevante para el individuo.

El objetivo de este estudio es explorar si el desempleo constituye un fenómeno con características semejantes al sufrido en un proceso de duelo. Con esta finalidad se adapta el Inventario Texas Revisado de Duelo al contexto de pérdida de empleo, analizando la relación existente entre determinadas variables sociodemográficas y las puntuaciones en intensidad de duelo, así como el empleo de determinadas estrategias de afrontamiento más o menos adaptativas. Estas relaciones podrán ser indicativas de determinadas evidencias de validez 
del uso de este instrumento en un contexto novedoso.

\section{Método}

\section{Participantes}

Los participantes de este estudio son 217 usuarios del Servicio Público de Empleo de Andalucía, procedentes del Centro de Orientación Laboral de la ciudad de Huelva. El muestreo fue sistemático, ofreciendo la participación en el estudio a todos los usuarios que accedían al centro durante los meses de marzo y octubre de 2014, aunque no todos los usuarios finalmente accedieron a colaborar en la investigación. De los participantes, el $44.7 \%$ son varones y $51.3 \%$ mujeres $\left(\chi^{2}=2.92\right.$, y $\left.p=.198\right)$, españoles en todos los casos, con edades entre 22 y 57 años $(M=37.12, D T=6.37)$, con nivel de formación en su mayoría básico (56.8\%; nivel medio $23.2 \%$ y nivel superior $16.8 \%$ ) y un tiempo medio en desempleo de 28 meses ( $D T=12.81)$.

\section{Instrumentos}

Para la evaluación de la intensidad de duelo se partió del Inventario de Duelo Texas Revisado (The Texas Revised Inventory of Grief, ITRD, Faschingbauer et al., 1987), adaptado al castellano por García-García y Landa (2001). La prueba original consta de dos escalas tipo Likert de cinco puntos: una de 8 ítems (parte I: duelo agudo), y otra de 13 ítems (parte II: duelo actual). Las puntuaciones más elevadas son indicativas de una mayor intensidad de duelo. Se adaptaron los ítems para que el contexto de pérdida de empleo. Para la adaptación de los ítems al contexto de desempleo se utilizó un método similar al realizado en las adaptaciones en un idioma diferente (Muñiz, Elosua, \& Hambleton, 2013), realizando una traducción doble. El resultado de dicha adaptación se muestra en el Anexo 1, que mantiene la estructura de dos escalas (parte I, duelo pasado: ítems 1 al 8; parte II, duelo actual: ítems 9 al 21). Para su administración se utilizó un formato de autoinforme, siguiendo las recomendaciones de la prueba original en el contexto de duelo por pérdida.

Para identificar las fases de duelo el manual de la prueba original establece cuatro tipos de duelo: duelo prolongado, puntuaciones por encima del P50 en ambas subescalas; duelo resuelto, puntuaciones por encima de P50 en agudo pero por debajo en actual; duelo ausente, puntuaciones inferiores a P50 en ambas subescalas; duelo retardado, puntuaciones por encima del P50 en duelo actual, pero bajo en duelo agudo. La fiabilidad de las puntuaciones estimada por el coeficiente alfa de Cronbach en su versión original fue de .77 para la parte I y de .86 para la parte II (García-García \& Landa, 2001).

En el estudio de evidencias de validez se incluyó la evaluación de las estrategias de afrontamiento por medio del COPE-28 (Crespo \& Cruzado, 1997), compuesto por 28 ítems que se agrupan de dos en dos dando lugar a 14 estrategias de afrontamiento: Afrontamiento activo, Planificación, Apoyo social emocional, Apoyo social instrumental, Religión, Reinterpretación positiva, Aceptación, Negación, Humor, Autodistracción, Autoinculpación, Desconexión conductual, Desahogo emocional y Uso de sustancias. Se estimó el alfa de Cronbach para cada factor obteniendo valores entre $.71 \mathrm{y}$ .82 .

Se incluyó también la evaluación de la ansiedad con el Inventario de Ansiedad de Beck (BAI; Beck \& Steer, 2011), adaptado al castellano (Sanz \& Navarro, 2003). Es un instrumento específicamente diseñado para evaluar la ansiedad prolongada y está menos contaminada por el contenido depresivo. Está conformada por 21 ítems en una escala tipo Likert de 0 a 3. Permite la agrupación de las personas en cuatro grupos: 0-7, sin ansiedad, 8-15, ansiedad leve, 16-25, ansiedad moderada y 26-63 ansiedad grave (Beck \& Steer, 2011). Las puntuaciones del cuestionario obtienen una alta consistencia interna con coeficientes alfa por encima de .80 (Sanz \& Navarro, 2003), siendo en nuestro muestra el coeficiente obtenido de .92 .

Finalmente se añadió un protocolo de recogida de información de variables sociodemográficas y laborales de interés para los objetivos del estudio. Entre las variables incluidas están la edad, sexo, nivel de estudios, el tiempo en desempleo, la antigüedad en su último empleo y el tiempo que le queda de recibir la prestación por desempleo, si son responsables de los ingresos familiares y si hay otro tipo de ingresos en la 
unidad familiar.

\section{Procedimiento}

La administración se realizó en el Centro de Orientación Laboral del Servicio Público de Empleo en la ciudad de Huelva. Los cuestionarios fueron administrados de forma individual en una sala independiente. Se aseguró la confidencialidad de los datos, y se informó de la voluntariedad de la participación en el estudio, obteniendo el consentimiento informado de cada uno de los participantes. Se descartó aquellos cuestionarios que presentaban datos faltantes, que supusieron el $2 \%$ de los cuestionarios recabados inicialmente.

En primer lugar se realizó un análisis de los ítems y de la fiabilidad de las puntuaciones de cada una de las escalas del instrumento con el coeficiente alfa de Cronbach. Dada que es la primera ocasión que se administra la adaptación de prueba se decidió realizar un análisis factorial exploratorio con el método máxima verosimilitud y rotación oblicua para analizar la estructura del Inventario Texas Revisado de Duelo aplicado en un contexto de desempleo. Para el cálculo de la solución factorial se obtuvo la matriz de correlaciones policóricas con STATA 13, realizando el resto del análisis con SPSS 20.

Seguidamente, con el objetivo aportar evidencias de validez e identificar grupos homogéneos de desempleados con diferentes niveles de duelo presente y pasado, se efectuó un análisis de conglomerados con los ítems del Inventario Texas Revisado de Duelo. Se utilizó el análisis de conglomerados de dos fases, utilizando el criterio bayesiano de Schwarz para determinar el número de conglomerados extraídos. Una vez establecidos los grupos, se contrastó la existencia de diferencias estadísticamente significativas entre ellos mediante pruebas $t$ para aquellas variables métricas y mediante pruebas de Chi-cuadrado para las variables categóricas. Este conjunto de análisis permiten la obtención de evidencias de validez de criterio, relacionando las puntuaciones del instrumento con otras variables sociodemográficas, laborales y psicológicas.

\section{Resultados}

\section{Fiabilidad y estructura factorial de la escala}

El análisis descriptivo inicial de los ítems se presenta en la Tabla 1, donde se observan valores de sesgo y curtosis adecuados $(<|2|)$, tanto en las puntuaciones de cada ítem como en el total de cada escala. Las correlaciones ítem-total se obtuvieron para cada una de las escalas, encontrando valores en un rango de $r=.454$ (ítem 4) y $r=.753$ (ítem 9). Realizado el análisis de fiabilidad de consistencia interna en nuestra, obtuvimos los valores del coeficiente alfa de .85 para la parte I y de .90 para la parte II, obteniendo un valor de .94 para la escala global.

Los comprobaciones previas $\left(\mathrm{KMO}=0.88 ; \chi^{2}\right.$ Bartlett=993.08; $p<.001)$ muestran que el análisis factorial se puede realizar. La inspección del gráfico de sedimentación, así como el análisis paralelo y el análisis de autovalores menores que 1 determinan una solución de dos factores que explican el $59.7 \%$ de la varianza. En la tabla 2 se muestran las cargas factoriales de cada uno de los ítems a los factores obtenidos. Todos los ítems presentan cargas más elevadas en el factor que corresponde según la construcción de la prueba, es decir los ítems del 1 al 8 tienen mayor peso en el factor 2 (que denominamos duelo actual) y los ítems 9 al 21 en el factor 1 (que denominamos duelo agudo), a excepción del ítem 4 , que muestra cargas factoriales similares en los dos factores. Se obtiene una distribución de cargas similar a lo encontrado en la versión original de Faschingbauer (1981) para duelo por pérdida de un ser querido.

\section{Análisis de evidencias de validez}

Con objeto de obtener ciertas evidencias de validez exploramos las relaciones entre las puntuaciones en intensidad de duelo y variables sociolaborales que según la literatura previa deben estar relacionadas con el duelo por desempleo. Para ello se realiza en primer lugar un análisis de conglomerados de dos fases, que ofreció la mejor solución extrayendo dos conglomerados (C1 y C2), con un $43.3 \%$ y un $56.7 \%$ de las personas en cada grupo respectivamente. En las Tablas 3 y 4 se muestran las comparaciones entre ambos conglomerados en las variables analizadas. 
Tabla 1. Análisis descriptivo de las puntuaciones en cada ítem y puntuaciones de cada escala

\begin{tabular}{ccccccc}
\hline & M & DT & Sesgo & Curtosis & $\begin{array}{c}\text { Correlacion } \\
\text { ítem-total }\end{array}$ & $\begin{array}{c}\text { Fiabildad si } \\
\text { se elimina }\end{array}$ \\
\hline Ítem 1 & 2.04 & 1.46 & 1.01 & -0.41 & .507 & .838 \\
Ítem 2 & 2.90 & 1.41 & 0.02 & -1.31 & .553 & .834 \\
Ítem 3 & 1.84 & 1.28 & 1.20 & 0.15 & .551 & .834 \\
Ítem 4 & 3.89 & 1.28 & -0.93 & -0.06 & .454 & .845 \\
Ítem 5 & 3.48 & 1.40 & -0.40 & -1.17 & .693 & .815 \\
Ítem 6 & 2.62 & 1.43 & 0.38 & -1.18 & .715 & .813 \\
Ítem 7 & 3.60 & 1.48 & -0.51 & -1.03 & .561 & .833 \\
Ítem 8 & 3.59 & 1.43 & -0.59 & -0.99 & .648 & .821 \\
Ítem 9 & 3.17 & 1.53 & -0.32 & -1.38 & .753 & .897 \\
Ítem 10 & 3.77 & 1.31 & -0.92 & -0.39 & .666 & .901 \\
Ítem 11 & 3.10 & 1.44 & -0.13 & -1.36 & .735 & .898 \\
Ítem 12 & 4.57 & .79 & -1.08 & 1.88 & .358 & .912 \\
Ítem 13 & 3.91 & 1.19 & -0.99 & 0.19 & .696 & .900 \\
Ítem 14 & 3.82 & 1.18 & -1.01 & 0.32 & .733 & .898 \\
Ítem 15 & 2.70 & 1.56 & 0.24 & -1.47 & .730 & .898 \\
Ítem 16 & 2.38 & 1.40 & 0.46 & -1.17 & .570 & .905 \\
Ítem 17 & 3.50 & 1.41 & -0.65 & -0.84 & .648 & .902 \\
Ítem 18 & 4.12 & 1.27 & -1.30 & 0.66 & .445 & .910 \\
Ítem 19 & 3.09 & 1.41 & -0.24 & -1.18 & .597 & .904 \\
Ítem 20 & 2.76 & 1.43 & 0.07 & -1.40 & .683 & .900 \\
Ítem 21 & 3.57 & 1.49 & -0.62 & -0.90 & .509 & .908 \\
Puntuación parte 1 & 20.40 & 8.08 & 0.46 & -0.51 & & \\
Puntuación parte 2 & 36.40 & 11.80 & 0.26 & -0.65 & & \\
\hline
\end{tabular}

En relación a los sentimientos en el pasado (ITRD parte 1), el conglomerado 1 obtiene una puntuación media mayor $(M=27.17, D T=6.03)$ que el segundo conglomerado $(M=15.40$, $D T=5.32$ ), siendo las diferencias significativas (ITRD parte1: $t_{215}=11.18, p<.000, d=1.52$ ). De la misma forma, hay diferencias significativas en la puntuación referida a los sentimientos expresados en la actualidad (ITRD parte2: $t_{215}=10.23$, $p=.000, d=1.39$ ), siendo el conglomerado 1 el que tiene una media más alta $(M=44.84, D T=8.18)$.

En la variable edad, no se observan diferencias significativas entre ambos conglomerados, $\left(t_{215}=1.33, p=.185, d=0.18\right)$, con medias $37.43 \quad(D T=5.89)$ y $35.61 \quad(D T=7.74)$ respectivamente. Del mismo modo, los meses de desempleo $(C 1: M=32.12, \quad D T=24.92 ; y \quad C 2$ : $M=22.19, \quad D T=16.81$ ), tampoco muestran diferencias significativas entre ambos conglomerados $\left(t_{215}=2.19, p=.031, d=0.29\right)$.

La Tabla 4 muestra las diferencias entre ambos conglomerados atendiendo a las variables categóricas. El porcentaje de hombres en cada grupo es similar $\left(\chi^{2}=0.45, p=.253\right)$. Tampoco es significativa la relación entre el nivel de los estudios cursados y el nivel de duelo que presentan los sujetos $\left(\chi^{2}{ }_{l}=2.47, p=.479\right)$.

El primer conglomerado presenta un porcentaje más alto de responsables de los ingresos familiares $(55.0 \%$ de responsables de los ingresos en el conglomerado 1 frente al $28.9 \%$ en el conglomerado 2) $\left(\chi_{1}^{2}=11.37, p=.003\right)$. También se encuentra una diferencia significativa entre aquellos que tienen una fuente alternativa de ingresos en la unidad familiar (C1: 47\%, C2: $67.2 \%)\left(\chi_{l}^{2}=3.92\right.$, y $\left.p=.048\right)$. Adicionalmente, se observa que, en el conglomerado 1 se encuentra un $53.1 \%$ de sujetos con ansiedad grave respecto al $28.4 \%$ en el conglomerado 2. Esta diferencia es significativa $\left(\chi^{2}=13.08\right.$ y $\left.p=.001\right)$.

Atendiendo a las fases de duelo establecidas en la prueba original (García-García \& Landa, 2001), según el patrón de respuestas las dos partes del ITRD, un $21.2 \%$ de los participantes tienen duelo ausente, un $10.7 \%$ duelo retardado, un $12.2 \%$ duelo resuelto, aunque la mayor parte de 
Tabla 2. Estructura factorial del cuestionario

\begin{tabular}{ccc}
\hline \multirow{2}{*}{ Ítems } & \multicolumn{2}{c}{ Dimensiones } \\
\cline { 2 - 3 } & 1 & 2 \\
\hline Ítem 1 & .102 & .667 \\
Ítem 2 & .053 & .720 \\
Ítem 3 & .162 & .674 \\
Ítem 4 & .307 & .440 \\
Ítem 5 & .281 & .713 \\
Ítem 6 & .275 & .759 \\
Ítem 7 & .309 & .572 \\
Ítem 8 & .292 & .674 \\
Ítem 9 & .699 & .406 \\
Ítem 10 & .692 & .233 \\
Ítem 11 & .687 & .302 \\
Ítem 12 & .434 & .095 \\
Ítem 13 & .675 & .239 \\
Ítem 14 & .752 & .235 \\
Ítem 15 & .688 & .381 \\
Ítem 16 & .620 & .150 \\
Ítem 17 & .718 & .143 \\
Ítem 18 & .533 & .081 \\
Ítem 19 & .617 & .246 \\
Ítem 20 & .692 & .268 \\
Ítem 21 & .597 & .091 \\
\hline
\end{tabular}

Método de extracción: Máxima verosimilitud. Método de rotación: Normalización Varimax con Kaiser

los desempleados presentan un duelo prolongado $53.9 \%$. En cuanto a la distribución de las fases de duelo según los conglomerados, encontramos que el primer conglomerado está más asociado a personas con duelo prolongado $(93.1 \%$ frente a $26.9 \%$ en el C2) $\left(\chi^{2}=56.78\right.$ y $\left.p=.000\right)$.

Las pruebas referidas a las diferentes estrategias de afrontamiento se muestran en la Tabla 4. Encontramos diferencias significativas entre conglomerados en las estrategias de autoinculpación $\quad\left(t_{215}=3.80, \quad p<.001, \quad d=0.51\right)$, desconexión $\quad\left(t_{215}=3.01, \quad p=.003, \quad d=0.41\right)$, desahogo $\quad\left(t_{215}=2.57, \quad p=.011, \quad d=0.35\right)$, autodistracción $\left(t_{215}=2.19, \quad p=.030, d=0.29\right), \quad \mathrm{y}$ negación $\quad\left(t_{215}=3.38\right.$ y $\left.p=.001, \quad d=0.46\right) . \quad \mathrm{El}$ conglomerado 1 se relaciona con puntuaciones medias mayores en todas estas estrategias evitativas (ver Tabla 4). Las estrategias de planificación mostraron puntuaciones medias mayores en el grupo 1, $(C 1: M=6.03, D T=1.19$; $C 2: M=5.58, D T=1.50)$ aunque estas diferencias no resultaron significativas $\left(t_{215}=1.76, p=.081\right.$, $d=0.24$ ). Como se puede observar en la tabla las diferencias entre las medias de las demás estrategias no son significativas.

\section{Discusión}

El presente trabajo aplica el Inventario de Texas Revisado de Duelo (ITRD) por primera vez al contexto de pérdida de empleo. Se obtuvo una fiabilidad de consistencia interna de .85 y de .90 en cada una de las partes, mostrando que las respuestas de las personas a este cuestionario en este contexto son consistentes, resultados que mejoran los encontrados en García-García y Landa (2001) con la prueba original. En cuanto a la estructura factorial del instrumento aplicado en un contexto de pérdida de empleo, se obtiene una estructura de dos factores y una distribución de cargas factoriales de cada factor que replican lo obtenido en la versión original (Faschingbauer, 1981). El ítem 4 en estudios anteriores también ha mostrado un funcionamiento diferente, con cargas factoriales repartidas (García-García, Landa Petralanda, Trigueros Manzano, \& Gaminde Inda, 2005; Samper Lucena, 2011). Estos resultados nos hacen pensar en la aplicabilidad de este instrumento para otro tipo de pérdida como es la pérdida de empleo, no solo por fallecimiento. Los estudios realizados sobre la versión adaptada al español del ITRD por García-García y Landa (2001), encuentran que las diferencias en las variables sexo y edad no son significativas para el duelo por pérdida, algo que nuestros resultados apoyan, suponiendo este resultado un primer indicio o evidencia de validez del instrumento en el contexto de pérdida de empleo. Los resultados del análisis de conglomerados muestran un grupo de desempleados con una mayor intensidad de duelo que coincide con personas que llevan más tiempo en desempleo y que tuvieron un trabajo de mayor antigüedad. Álvaro, Guedes, Garrido, Figueiredo y Campos (2012) señalan la prolongación del período de desempleo como uno de los factores más investigados. La duración del desempleo incide sobre la salud con un marcado deterioro en la salud mental (Del Pozo, Ruiz, Pardo, \& San Martín, 2002). De acuerdo a Harvey y Weber (1998), los resultados apuntan a unas primeras evidencias respecto a que el esfuerzo en la elaboración del duelo se acentúa cuanto más próxima y duradera es la relación establecida. 
Tabla 3. Estadísticos descriptivos y diferencia entre conglomerados en las variables métricas

\begin{tabular}{ccccccc}
\hline Variables & Grupo & $\mathrm{M}$ & $\mathrm{DT}$ & $\mathrm{t}$ & $p$ & $\mathrm{~d}$ \\
\hline $\begin{array}{c}\text { Texas. Sentimientos } \\
\text { pasados }\end{array}$ & $\mathrm{C} 1$ & 27.17 & 6.03 & 11.18 & .000 & 1.52 \\
$\begin{array}{c}\text { Texas. Sentimientos } \\
\text { actuales }\end{array}$ & $\mathrm{C} 1$ & 44.84 & 8.18 & 10.23 & .000 & 1.39 \\
& $\mathrm{C} 2$ & 28.36 & 9.04 & & & \\
Edad & $\mathrm{C} 1$ & 37.43 & 5.89 & 1.33 & .185 & 0.18 \\
& $\mathrm{C} 2$ & 35.61 & 7.74 & & & \\
$\begin{array}{c}\text { Meses en desempleo } \\
\text { (1) }\end{array}$ & $\mathrm{C} 1$ & 32.12 & 24.92 & 2.19 & .031 & 0.29 \\
$\begin{array}{c}\text { Meses antigüedad } \\
\text { ultimo empleo }\end{array}$ & $\mathrm{C} 1$ & 58.86 & 53.86 & 2.27 & .025 & 0.30 \\
& $\mathrm{C} 2$ & 36.16 & 53.98 & & & \\
$\begin{array}{c}\text { Tiempo que le queda de } \\
\text { recibir ayuda }\end{array}$ & $\mathrm{C} 1$ & 3.69 & 3.31 & 0.45 & .651 & 0.06 \\
\hline
\end{tabular}

C1: Conglomerado 1 (n=94); C2: conglomerado $2(\mathrm{n}=123)$

En este sentido, se encuentran las aportaciones referidas en la teoría del desarrollo vocacional (Super, Savickas, \& Super, 1996). Desde este encuadre, en las etapas de establecimiento y mantenimiento del empleo se establece un vínculo personal y emocional con la ocupación. Por tanto, una de las aportaciones más relevantes de este estudio es que describe un duelo más intenso cuanto más estable y duradera fue la situación laboral anterior a la pérdida.

No encontramos diferencias en relación al nivel de estudios ni respecto el tiempo que les queda por recibir algún tipo de ayuda por desempleo. Este resultado refuerza el argumento de que el desempleo está relacionado con un proceso del ámbito emocional. Siguiendo a Salanova, Osca, Peiró y Sarcerni (1991), el trabajo no tiene un significado exclusivamente económico, sino que lleva consigo una serie de creencias que le dan significado e identidad.

También es una realidad que una disminución de los ingresos conlleva una serie de consecuencias. De acuerdo a Jacobson, LaLonde y Sullivan (1993), el desempleo repercute sobre la seguridad económica de la unidad familiar, lo que explica que hayamos encontrado diferencias referidas a la responsabilidad de los ingresos familiares y la existencia o no de apoyos económicos (ayudas, prestaciones, pensiones). Así, las personas con mayores dificultades económicas se encuentran en el grupo que experimentan el duelo de forma más intensa. Parece evidente que el factor económico también podría considerarse una variable que incrementara el riesgo de padecer algún deterioro de la salud en el desempleado así como para las personas que se encuentran a su cargo.

En cuanto a las estrategias de afrontamiento, encontramos que las personas que experimentan un duelo más intenso utilizan en mayor medida estrategias basadas en la negación, autoinculpación, autodistracción, y desconexión. En este sentido, el estilo de afrontamiento basado en la evitación (negación, autodistracción, 
Tabla 4. Porcentajes y diferencia entre conglomerados de las variables categóricas.

\begin{tabular}{|c|c|c|c|c|}
\hline Variables & Grupo & Porcentaje & $\chi^{2}$ & $\mathrm{p}$ \\
\hline \multirow{2}{*}{ Hombres } & $\mathrm{C} 1$ & 61.8 & 0.45 & .253 \\
\hline & $\mathrm{C} 2$ & 60.9 & & \\
\hline \multirow{2}{*}{ Estudios básicos } & $\mathrm{C} 1$ & 44.1 & 2.47 & .479 \\
\hline & $\mathrm{C} 2$ & 43.2 & & \\
\hline \multirow{2}{*}{$\begin{array}{l}\text { Responsable de los ingresos } \\
\text { familiares }\end{array}$} & $\mathrm{C} 1$ & 55.0 & 11.37 & .003 \\
\hline & $\mathrm{C} 2$ & 28.9 & & \\
\hline \multirow{2}{*}{$\begin{array}{c}\text { Tienen otros ingresos en la unidad } \\
\text { familiar }\end{array}$} & $\mathrm{C} 1$ & 47.0 & 3.92 & .048 \\
\hline & $\mathrm{C} 2$ & 67.2 & & \\
\hline \multirow[t]{2}{*}{ Ansiedad grave } & $\mathrm{C} 1$ & 53.1 & 13.08 & .001 \\
\hline & $\mathrm{C} 2$ & 28.4 & & \\
\hline \multirow{2}{*}{ Duelo ausente } & $\mathrm{C} 1$ & 0 & 56.78 & .000 \\
\hline & $\mathrm{C} 2$ & 39.3 & & \\
\hline \multirow{2}{*}{ Duelo retrasado } & $\mathrm{C} 1$ & 2.9 & & \\
\hline & $\mathrm{C} 2$ & 13.4 & & \\
\hline \multirow{2}{*}{ Duelo resuelto } & $\mathrm{C} 1$ & 4.0 & & \\
\hline & $\mathrm{C} 2$ & 20.4 & & \\
\hline \multirow{2}{*}{ Duelo prolongado } & $\mathrm{C} 1$ & 93.1 & & \\
\hline & $\mathrm{C} 2$ & 26.9 & & \\
\hline
\end{tabular}

C1: Conglomerado $1(\mathrm{n}=94) ; \mathrm{C} 2$ : conglomerado $2(\mathrm{n}=123)$

Tabla 5. Estadísticos descriptivos y diferencia entre conglomerados de las estrategias de afrontamiento

\begin{tabular}{|c|c|c|c|c|c|c|}
\hline Conglomerados & Grupo & $\mathrm{M}$ & DT & $\mathrm{t}$ & $\mathrm{p}$ & $\mathrm{d}$ \\
\hline \multirow{2}{*}{ Afrontamiento activo } & $\mathrm{C} 1$ & 6.41 & 1.16 & 0.76 & .445 & 0.10 \\
\hline & $\mathrm{C} 2$ & 6.22 & 1.46 & & & \\
\hline \multirow{2}{*}{ Planificación } & $\mathrm{C} 1$ & 6.03 & 1.19 & 1.76 & .081 & 0.24 \\
\hline & $\mathrm{C} 2$ & 5.58 & 1.50 & & & \\
\hline \multirow{2}{*}{ Apoyo emocional } & $\mathrm{C} 1$ & 5.23 & 1.51 & 0.29 & .769 & 0.03 \\
\hline & $\mathrm{C} 2$ & 5.32 & 1.68 & & & \\
\hline \multirow{2}{*}{ Apoyo social } & $\mathrm{C} 1$ & 5.68 & 1.37 & 1.26 & .210 & 0.17 \\
\hline & C2 & 5.35 & 1.46 & & & \\
\hline \multirow{2}{*}{ Religión } & $\mathrm{C} 1$ & 3.27 & 1.63 & 0.03 & .975 & 0.00 \\
\hline & $\mathrm{C} 2$ & 3.26 & 1.72 & & & \\
\hline \multirow{2}{*}{ Reevaluación } & $\mathrm{C} 1$ & 5.29 & 1.43 & 0.19 & .850 & 0.03 \\
\hline & $\mathrm{C} 2$ & 5.35 & 1.83 & & & \\
\hline \multirow{2}{*}{ Aceptación } & $\mathrm{C} 1$ & 5.35 & 1.69 & 0.04 & .962 & 0.00 \\
\hline & $\mathrm{C} 2$ & 5.36 & 1.61 & & & \\
\hline \multirow{2}{*}{ Negación } & $\mathrm{C} 1$ & 4.11 & 1.62 & 3.38 & .001 & 0.46 \\
\hline & $\mathrm{C} 2$ & 3.19 & 1.36 & & & \\
\hline \multirow{2}{*}{ Humor } & $\mathrm{C} 1$ & 3.70 & 1.81 & 0.97 & .332 & 0.13 \\
\hline & $\mathrm{C} 2$ & 3.41 & 1.47 & & & \\
\hline \multirow{2}{*}{ Autodistracción } & $\mathrm{C} 1$ & 5.01 & 1.56 & 2.19 & .030 & 0.29 \\
\hline & $\mathrm{C} 2$ & 4.41 & 1.43 & & & \\
\hline \multirow{2}{*}{ Autoinculpación } & $\mathrm{C} 1$ & 4.07 & 1.43 & 3.80 & .000 & 0.51 \\
\hline & $\mathrm{C} 2$ & 3.00 & 1.12 & & & \\
\hline \multirow{2}{*}{ Desconexión } & $\mathrm{C} 1$ & 3.31 & 1.40 & 3.01 & .003 & 0.41 \\
\hline & $\mathrm{C} 2$ & 2.64 & 1.00 & & & \\
\hline \multirow{2}{*}{ Desahogo } & $\mathrm{C} 1$ & 4.31 & 1.25 & 2.57 & .011 & 0.35 \\
\hline & $\mathrm{C} 2$ & 3.64 & 1.49 & & & \\
\hline \multirow{2}{*}{ Sustancias } & $\mathrm{C} 1$ & 2.56 & 1.17 & 0.30 & .766 & 0.04 \\
\hline & $\mathrm{C} 2$ & 2.63 & 1.13 & & & \\
\hline
\end{tabular}


desconexión) es propio de la pérdida de esperanza ante la dificultad que supone la búsqueda de empleo en un periodo de crisis (McLoyd, Jayaratne, Ceballo, \& Borquez, 1994). Gondim, Álvaro, Luna, Oliveira y Souza (2010) relacionan los procesos de atribución causal y la depresión, la autoestima, el locus de control o las actitudes frente al mercado de trabajo. Los resultados de estos estudios vienen a constatar que la atribución de la falta de empleo a causas internas, globales y estables tiende a estar asociada a déficits depresivos y que la motivación para buscar empleo depende de un locus de control interno y atribuciones internas sobre el éxito o fracaso en el mercado de trabajo. Estos resultados se pueden interpretar como evidencias de validez del uso de este instrumento en un contexto de pérdida de empleo.

El presente trabajo abre nuevas posibilidades en el estudio de las consecuencias psicológicas del desempleo, permitiendo relacionarlas con elementos idiosincráticos de las teorías del duelo. De esta forma, se puede superar la visión sobre consecuencias psicológicas estáticas para cada situación e individuo en desempleo, por una visión más dinámica y realista, que asigne diferentes consecuencias e intensidad de las mismas en función, de la tipología, intensidad y momento del duelo por la pérdida de empleo en el que se encuentre la persona. Desde el punto de vista aplicado, se estima la necesidad de incorporar estos resultados a la intervención que desde los servicios sociales y de empleo se realiza con las personas desempleadas. La visión del desempleo como pérdida, permitirá superar las estrategias llevadas a cabo hasta la fecha para el aumento de la empleabilidad de estas personas, y que suelen ser de carácter generalista y uniforme, finalizando con la prescripción de ciertos recursos de orientación y formación, sin tener en cuenta si el momento y/o la intensidad del proceso de duelo por pérdida de empleo seguido por esa persona se adecúa o no al recurso previsto.

El estudio presentado se realiza en coincidencia con la importante crisis de empleo sufrida por numerosos países del sur de Europa, resultando de especial virulencia en España, por lo que se evidencia la importante pertinencia y vigencia de sus planteamientos. No obstante, sería conveniente la realización de estudios similares Revista Iberoamericana de Diagnóstico y Evaluación - e Avaliação Psicológica. RIDEP · Nº42 · Vol.2 · 111-124 · 2016 con poblaciones desempleadas de otros ámbitos geográficos, tanto dentro como fuera de las fronteras españolas. Sería de interés la comparación de estrategias de afrontamiento y la intensidad del duelo por la pérdida de empleo relacionándolos con diversos contextos socioculturales, así como con las diferentes consideraciones acerca del valor que se le otorga al trabajo en unas culturas con respecto a otras.

Otras líneas de investigación futuras, apuntarían a estudios longitudinales que permitirán explicar el comportamiento en posteriores puestos de trabajo de desempleados evaluados con diversos grados de intensidad de duelo por pérdida de empleo. De este modo se podrían establecer interesantes relaciones con niveles de salud laboral, estrés o engagement, entre otros.

\section{Referencias}

Afonso, R. M., \& Poeschl, G. (2006). Representaciones del impacto de la situación de desempleo en las prácticas familiares. Revista de Psicología Social, 21(3), 241-258.

Álvaro, J., \& Garrido A. (2003). Desempleo, salud y exclusión social. Madrid: Siglo XX.

Álvaro, J. L., Guedes, S., Garrido, A., Figueiredo, A., \& Campos, M. (2012). Desempleo y bienestar psicológico en Brasil y España: Un estudio comparativo. Revista Psicologia: Organizações e Trabalho, 12(1), 5-16

American Psychiatric Association (APA) (2013). Guía de consulta de los criterios diagnósticos del DSM-V. Arlington VA: Asociación Americana de Psiquiatría.

Beck, A. T., \& Steer, R. A. (2011). Manual BAI. Inventario de Ansiedad de Beck (Adaptación española de Sanz, J.). Madrid: Pearson Educación.

Blanch, J. M. (1990). Del viejo al nuevo paro: Un análisis psicológico y social. Barcelona: PPU.

Bowlby, J. (1980). El apego y la pérdida: La pérdida. Barcelona: Paidós.

Buendía, J. (1990). Psicopatología del desempleo. Anales de Psicología, 6(1), 21-36.

Buendía, J. (2010). El impacto psicológico del desempleo. Murcia: Universidad de Murcia.

Cáceres, C., Manhey, C., \& Vidal C. (2009). 
Separación, pérdida y duelo de la pareja: Reflexiones imprescindibles para una terapia de divorcio. Revista de Familias y Terapias, 2(7), 41-60.

Carmona, C., Cely, J., Nemeguen, R., \& Sanabria, W. (2008). Características del proceso de duelo por pérdida de actividad laboral que se presenta en población en proceso de prejubilación del Dane. Umbral Científico, 12, 101-116.

Crespo, M., \& Cruzado, J. A. (1997). La evaluación del afrontamiento: Adaptación española del cuestionario COPE con una muestra de estudiantes universitarios. Análisis y Modificación de Conducta, 23(92), 797-830.

Del Pozo, J. A., Ruiz, M. A., Pardo, A., \& San Martín, R. S. (2002). Efectos de la duración del desempleo entre los desempleados. Psicothema, 14(2), 440-443.

Faschingbauer, T. R. (1981). Texas Revised Inventory of Grief manual. Houston: Honeycomb Publishing.

Faschingbauer, T., Zisook, S., \& DeVaul, R. (1987). The Texas Revised Inventory of Grief. In S. Zisook (Ed.), Biopsychosocial aspects of bereavement (pp.111-124). Washington, DC: American Psychiatric Press.

Fernández, A., Rodríguez, B., \& Diéguez, M. (2006). Intervenciones sobre duelo. En P. Pérez-Salas (Eds.). Trauma, culpa y duelo. Hacia una psicoterapia integradora. (pp. 447496). Bilbao: Desclée de Brouwer.

Fernández-Abascal, E. G. (1997). Estilos y estrategias de afrontamiento. [Styles and coping strategies]. En E.G. Fernández Abascal, F. Palmero, M. Chóliz \& F. Martínez (Eds.). Cuaderno de Prácticas de Motivación y Emoción, (pp. 189-206). Madrid: Pirámide.

García-García, J. A., \& Landa, V. (2001). ¿Es posible medir el duelo? Adaptación al castellano y validación del Inventario de Experiencias en Duelo (IED) y del Inventario Texas Revisado de Duelo (ITRD). Psiquiatria.com, 5 (1).

García-García, J. A., Landa Petralanda, V., Trigueros Manzano, M. C., \& Gaminde Inda, I. (2005). Inventario Texas Revisado de Duelo (ITRD): Adaptación al castellano, fiabilidad y validez. Atención Primaria, 35(7), 353-358.
Gillham, J. E., \& Seligman, M. E. P. (1999). Footsteps on the road to positive psychology. Behaviour Research and Therapy, 37, 163173.

Gondim, S. M. G., Álvaro, J. L., Luna, A. F., Oliveira, T., \& Souza, G. C. (2010). Atribuições de causas ao desemprego e valores pessoais. Estudos de Psicologia, 15, 309-317.

González-Calvo, V. (2005) El duelo migratorio. Revista del departamento de Trabajo Social, Facultad de Ciencias Humanas, Universidad Nacional de Colombia, 7, 77-97.

Harvey, J. H., \& Weber, A. L. (1998). Why there must be a psychology of loss. In J. H. Harvey (Ed.), Perspectives on loss: A sourcebook (pp. 319-329). Philadelphia: Brunner/Mazel.

Hegewald, M. J., \& Crapo, R. O. (2007). Socioeconomic status and lung function. Chest, 132(5), 1608-14.

Izquierdo-Rus, T., \& López-Martínez, O. (2013). Variables que afectan a la creatividad de las personas desempleadas. Anales de Psicología, 29(1), 103-107. http://dx.doi.org/10.6018/analesps.29.1.13810 1

Jacobson, L., LaLonde, R., \& Sullivan, D. (1993). Earning losses of displaced workers. American Economic Review, 83, 685-709.

Karsten, I., \& Moser, K. (2009). Unemployment impairs mental health: Meta-analysis. Journal of Vocational Behaviour, 74, 264-282.

Kübler-Ross, E. (1993). Sobre la muerte y los moribundos $\left(3^{a}\right.$ ed). Barcelona: Grijalbo.

Lazarus, R. S., \& Folkman, S. (1984). Estrés y procesos cognitivos. Barcelona: Martínez Roca.

Lugonés, M. (2001). El climaterio y el síndrome del nido vacío en el contexto sociocultural. Revista Cubana de Medicina General Integral, 17(2), 206-208.

Martínez Pérez, M., \& Sánchez Aragón, R. (2011). Evaluación Multimétodo de la Expresión Emocional. Revista Iberoamericana de Diagnóstico y Evaluación - e Avaliação Psicológica, 31(1), 11-35.

McLoyd, V., Jayaratne, T., Ceballo, R., \& Borquez, J. (1994). Unemployment and work interruption among African American single mother: Effects on parenting and adolescent Vol.2 $\cdot 111-124 \cdot 2016$ 
socioemotional functioning.

Child

Development, 65, 562-589.

Meléndez, J. C., Mayordomo, T., Sancho, P., \& Tomás, J. M. (2012). Coping strategies: Gender differences and development throughout life span. The Spanish Journal of Psychology, 15(3), 1089-1098. http://dx.doi.org/10.5209/rev_SJOP.2012.v15. n3.39399

Munera, P. (2013). El duelo por suicidio. Análisis del discurso de familiares y de profesionales de salud mental. Tesis doctoral, Universidad de Granada.

Muñiz, J., Elosua, P., \& Hambleton, R. K. (2013). Directrices para la traducción y adaptación de los tests: Segunda edición. Psicothema, 25(2), 151-157.

Neimeyer, R. (2002). Aprender de la pérdida. Una guía para afrontar el duelo. Barcelona: Paidós.

OMS. (2003). CIE10. Décima revisión de la Clasificación Internacional de las Enfermedades, Trastornos Mentales y del comportamiento de la OMS. Madrid: Meditor.

Ortego, M. C., \& Alconero, A. R. (2001).

El duelo. Enfermería Científica, 236-237, 46-51.

Prigerson, H. G., Shear, M. K., Jacobs S. C., Reynolds, C. F. 3rd, Maciejewski, P. K., Davidson J. R. T., et al. (1999). Consensus criteria for traumatic grief. A preliminary empirical test. British Journal of Psychiatry, 174, 67-73.

Salanova, M., Osca, M., Peiró, J. M., \& Sarcerni, M. (1991). Significado del trabajo en los jóvenes en la transición e incorporación al mercado laboral. Revista de Psicología General y Aplicada, 44(1), 113-125.

Samper Lucena, E. (2011). Adaptación psicométrica del Inventario de Texas Revisado de duelo (ITRD) en población militar española joven adulta. Sanidad militar, 67(3), 291-298.

Sánchez, R., \& Martínez. R. (2014). Causas y caracterización de las etapas del duelo romántico. Actas de Investigación Psicológica, 4(1), 1329-1343.

Sánchez-Aragón, R., \& Retana-Franco, B. (2013).

Evaluación tridimensional del duelo amoroso en México. Revista Iberoamericana de Diagnóstico y Evaluación - e Avaliação Psicológica, 36(2), 49-69.

Sanders, C. M. (1999). Risk factors in bereavement outcome. In M.S. Stroebe, W. Stroebe \& R. O. Hansson (Eds.), Handbook of bereavement: Theory, research and intervention (pp. 255-267). Cambridge: Cambridge University Press.

Sanders, C. M., Mauger, P. A., \& Strong, P. N. (1985). A manual for the Grief Experience Inventory. North Carolina: The Center for the Study of Separation and Loss

Sanz, J., \& Navarro, M. E. (2003). Propiedades psicométricas de una versión española del Inventario de Ansiedad de Beck (BAI) en estudiantes universitarios. Ansiedad y Estrés, 9, 59-84.

Siracusa, F., Quintana, F. C., Marfil, M. N. P., Caro, M. P. G., Río-Valle, J. S., \& Martínez, M. M. V. (2011). Actitudes y afrontamiento ante la muerte en padres de niños de primaria. Psicología conductual, 3, 627-642.

Stroebe, M. S., Folkman, S., Hansson, R. O., \& Schut, H. (2006). The prediction of bereavement outcome: Development of an integrative risk factor framework. Social Science \& Medicine, 63(9), 2440-2451. http://dx.doi.org/10.1016/j.socscimed.2006.06 .012

Stroebe, M., \& Schut, H. (1999). The dual process model of coping with bereavement: Rationale and description. Death Studies, 23(3), 197224.

Super, D. E.; Savickas, M. L., \& Super, C. M. (1996). The life-span, life-space approach to career. In D. Brown \& L. Brooks (Eds.). Career choice and development (pp. 122178). San Francisco: Jossey-Bass.

Tedeschi, R. G., \& Kilmer, R. P. (2005). Assessing strengths, resilience, and growth to guide clinical interventions. Professional Psychology: Research and Practice, 36(3), 230-237. http://dx.doi.org/10.1037/07357028.36.3.230

Tizón, J. L. (2004). Pérdida, pena, duelo. Vivencias, investigación y asistencia. Barcelona: Paidós. 
Villacieros, M., Serrano, I., Bermejo, J. C., Magaña, M., \& Carabias, R. (2014). Social support and psychological well-being as posible predictors of complicated grief in a cross-section of people in mourning. Anales de Psicología, 30 (3), 944-951. Disponible en http://dx.doi.org/10.6018/analesps.30.3.15469 1 
Anexo 1.

\section{INVENTARIO TEXAS REVISADO DE DUELO ADAPTADO AL CONTEXTO DE PÉRDIDA DE EMPLEO}

1. Tras la pérdida de trabajo me costaba relacionarme con algunas personas

2. Tras la pérdida de trabajo me costaba concentrarme en la búsqueda de empleo

3. Tras la pérdida de trabajo perdí el interés en mi familia, amigos y actividades fuera de casa

4. Tras la pérdida de trabajo tenía la necesidad de hacer las cosas que hacía cuando estaba trabajando

5. Después de la pérdida de trabajo estaba más irritable de lo normal

6. En los tres primeros meses después de la pérdida del trabajo me sentía incapaz de realizar mis actividades habituales

7. Me sentía furioso/a porque me había quedado sin trabajo

8. Tras la pérdida de trabajo me costaba trabajo dormir

9. Todavía tengo ganas de llorar cuando pienso en el trabajo

10. Todavía me pongo triste cuando pienso en el trabajo

11. No puedo aceptar la pérdida de mi trabajo

12. A veces echo mucho de menos ir a trabajar

13. Todavía me resulta doloroso traer a la memoria el recuerdo del trabajo

14. A menudo me quedo ensimismado pensando en mi trabajo

15. Lloro a escondidas cuando pienso en mi trabajo

16. Nada podrá ocupar jamás el lugar que ese trabajo ha dejado en mi vida

17. No puedo dejar de pensar en mi trabajo

18. Creo que no es justo que haya perdido el empleo

19. Las cosas y las personas que me rodean todavía me hacen recordar las cosas relacionadas con mi trabajo

20. Soy incapaz de aceptar la pérdida del empleo

21. A veces me invade la necesidad de estar trabajando otra vez en el mismo sitio 